\title{
Article \\ Noninvasive Magnetic-Marking-Based Flow Metering with Optically Pumped Magnetometers
}

\author{
Leonhard Schmieder ${ }^{1,2, *} \mathbb{D}$, Peter A. Koss ${ }^{1}{ }^{\mathbb{D}}$, Armin Lambrecht ${ }^{1}$ and Frank Kühnemann ${ }^{1} \mathbb{D}$ \\ 1 Fraunhofer IPM, Georges-Köhler-Allee 301, 79110 Freiburg im Breisgau, Germany; \\ peter.koss@ipm.fraunhofer.de (P.A.K.); armin.lambrecht@ipm.fraunhofer.de (A.L.); \\ frank.kuehnemann@ipm.fraunhofer.de (F.K.) \\ 2 Faculty of Mathematics and Physics, University of Freiburg, Hermann-Herder-Str. 3, \\ 79104 Freiburg im Breisgau, Germany \\ * Correspondence: leonhard.schmieder@ipm.fraunhofer.de; Tel.: +49-761-8857-277
}

check for

updates

Citation: Schmieder, L.; Koss, P.A.;

Lambrecht, A.; Kühnemann, F.

Noninvasive

Magnetic-Marking-Based Flow

Metering with Optically Pumped

Magnetometers. Appl. Sci. 2022, 12,

1275. https://doi.org/10.3390/

app12031275

Academic Editors: Arkadiusz Gola Izabela Nielsen and Patrik Grznár

Received: 6 December 2021

Accepted: 15 January 2022

Published: 25 January 2022

Publisher's Note: MDPI stays neutral with regard to jurisdictional claims in published maps and institutional affiliations.

Copyright: (C) 2022 by the authors. Licensee MDPI, Basel, Switzerland. This article is an open access article distributed under the terms and conditions of the Creative Commons Attribution (CC BY) license (https:// creativecommons.org/licenses/by/ $4.0 /)$.
Featured Application: Noninvasive inline flow-metering procedure with optically pumped magnetometers.

\begin{abstract}
We present a noninvasive procedure that measures the flow velocity of a fluid by using polarized hydrogen nuclei in the fluid. The measurement procedure is based on a time-of-flight method where magnetic information is applied on the fluid with a permanent magnet and an RF-pulse. In contrast to other methods, this magnetic-marking method works without tracers. The read-out of the magnetic information is performed by optically pumped magnetometers downstream. In order to function, the magnetometers have to be operated in a magnetic shield with magnetic field strengths lower than $100 \mathrm{nT}$, i.e., in the zero-to-ultra-low-field regime. In this regime, the magnetometers are capable of detecting induced magnetic signals of $10 \mathrm{pT}$ or less with an inline-flow setup. The results presented in this paper demonstrate the viability of optically pumped magnetometers for flow metering. The first metering results yielded an average accuracy of $3 \%$ at flow velocities between $13 \mathrm{~cm} / \mathrm{s}$ and $22.4 \mathrm{~cm} / \mathrm{s}$.
\end{abstract}

Keywords: ZULF-NMR; flow metering; OPM; time-of-flight measurement; noninvasive; tracer-free; calibration-free; inline measurement; volumetric flow

\section{Introduction}

Flow is one of the most important variables in industrial environments, and its precise measurement is the foundation of process automation. It is an established method in the production, infrastructure and process industry [1]. Electromagnetic flow metering (EMFM) is widely used for flow monitoring in research and industry [1]. However, it requires a minimum conductivity of $5 \mu \mathrm{S} / \mathrm{cm}$ of the fluid of interest. For nonconductive and multiphase media, which include non-ionized gases, ceramics, fuels or oils, there is no equivalent volumetric flow-metering technique [2].

NMR-based flow metering has proven to be a viable tool for multiphase flow detection in clinical applications and does not require a minimum conductivity [3,4]. Conventional high-field NMR measuring devices established on the market are rarely used in industry [5]. The difficult system integration and the associated costs limit its applicability [6]. In the high field NMR regime, strong alternating magnetic fields are a compromise between signal strength and field generation effort. For noninvasive flow metering through metal pipes, the reduced penetration depth of the radiofrequency (RF) signals is an additional obstacle [7].

For field strengths similar to the Earth's magnetic field or lower $(<50 \mu \mathrm{T})$, these disadvantages are significantly attenuated. Low magnetic fields are easy to generate, can be varied rapidly, and the low frequency RF-signals penetrate deeper through metallic 
enclosures such as pipes [7]. Initial research on low-field NMR techniques applied to flow metering is available [8,9]. This research has already demonstrated the feasibility of NMR-based flow measurement in the Earth's magnetic field using induction coils [10,11]. However, for a precise flow-metering method at field strengths below $50 \mu \mathrm{T}$, magnetometers are the better choice. In this field regime, signal frequencies are expected to be in the lower $\mathrm{kHz}$ to $\mathrm{Hz}$ range. For these frequencies, magnetometers show a higher signal-to-noise ratio compared to induction coils $[7,12]$.

While the most sensitive magnetometers were cryogenically cooled SQUID detectors for a long time, the first optically pumped magnetometers (OPM) are now commercially available. They allow measurements with comparable sensitivity at room temperature [13]. The working principle of an OPM is based on alkali atoms in a vapor cell, which are spin-polarized by a laser. The spin polarization of the atoms couples to the magnetic field to be measured and generates a measurable frequency [14].

Utilizing the high magnetic sensitivity of OPM also renders magnetic tracer particles obsolete. Magnetic tracer particles are ferromagnetic clusters of atoms or molecules. When mixed in a liquid, these particles differ from the solution in their magnetic properties. Thus, the determination of the fluid movement is facilitated by monitoring the positions of the magnetic particles [15].

The present research demonstrates a noninvasive tracer-free flow metering procedure, which uses an OPM operating in the zero-to-ultra-low-field (ZULF) regime as the signal detector. In a first step, the medium to be analyzed (here: water) is pre-polarized. Then, a resonant RF-pulse applies a magnetic time stamp to the medium by changing the water polarization locally. This time stamp can be detected with magnetometers such as OPM downstream. The time difference between pulsing and detecting indicates the flow velocity of the medium. In essence, the apparatus performs a time-of-flight measurement, which also makes the procedure calibration-free.

\section{Materials and Methods}

\subsection{Experimental Setup}

The experimental setup of the flow-metering apparatus and the orientation of the magnetic fields involved in the procedure is shown in Figures 1 and 2. The water reservoir consisted of a plastic syringe of $200 \mathrm{~mL}$ capacity. The syringe was operated by a syringe pump (Syringe Pump LA-800, Landgraf, Langenhagen, Germany), which can log pump rates digitally. For pre-polarization of the water sample the syringe was placed inside a $1 \mathrm{~T}$ permanent magnet (RHR-1T-50 Halbach magnet, BFLUX TECHNOLOGY, Dublin, Ireland). This way the whole volume of the syringe could be polarized before being pushed into the system. Pre-polarization was used to increase the magnetic signal for the OPM detector to a measurable level [7]. An interaction section and magnetic shielding (MS-2 Magnetic shield, Twinleaf LLC, Plainsboro Township, NJ, USA) containing magnetometers (Zero Field OPM, FieldLine Inc., Boulder, CO, USA.) were located downstream. The circuit ended in a water storage tank.

The interaction section consisted of a Helmholtz-coil (Ferronato ${ }^{\circledR}$-BHC-2, Serviciencia, S.L.U., Toledo, Spain), which was used as an RF coil, and racetrack coils. The racetrack coils served a dual function of spin guiding between Halbach and shield, and providing a constant ambient magnetic field necessary for RF pulsing. The presence of a magnetic field is necessary for RF pulsing because a manipulation of the water polarization is not possible otherwise. In the presence of a magnetic field, the nuclear spin energy level of the hydrogen atom is split in two equidistant energy levels. This is called the Zeeman effect. To induce a transition between the energy levels, which define the macroscopic polarization of the water, an RF pulse with the transition frequency of the hydrogen can be applied. This transition frequency is called Larmor precession $\omega_{L}$ and is defined as $\omega_{L}=\gamma B$. Here, $\gamma$ is the gyromagnetic ratio of the proton, which is roughly $42.577 \mathrm{MHz} / \mathrm{T}$, and $B$ is the strength of the ambient magnetic field. In the case of this experiment, the field generated by the racetrack coils was about $200 \mu \mathrm{T}$, which translates to an RF frequency of roughly $8 \mathrm{kHz}$. 


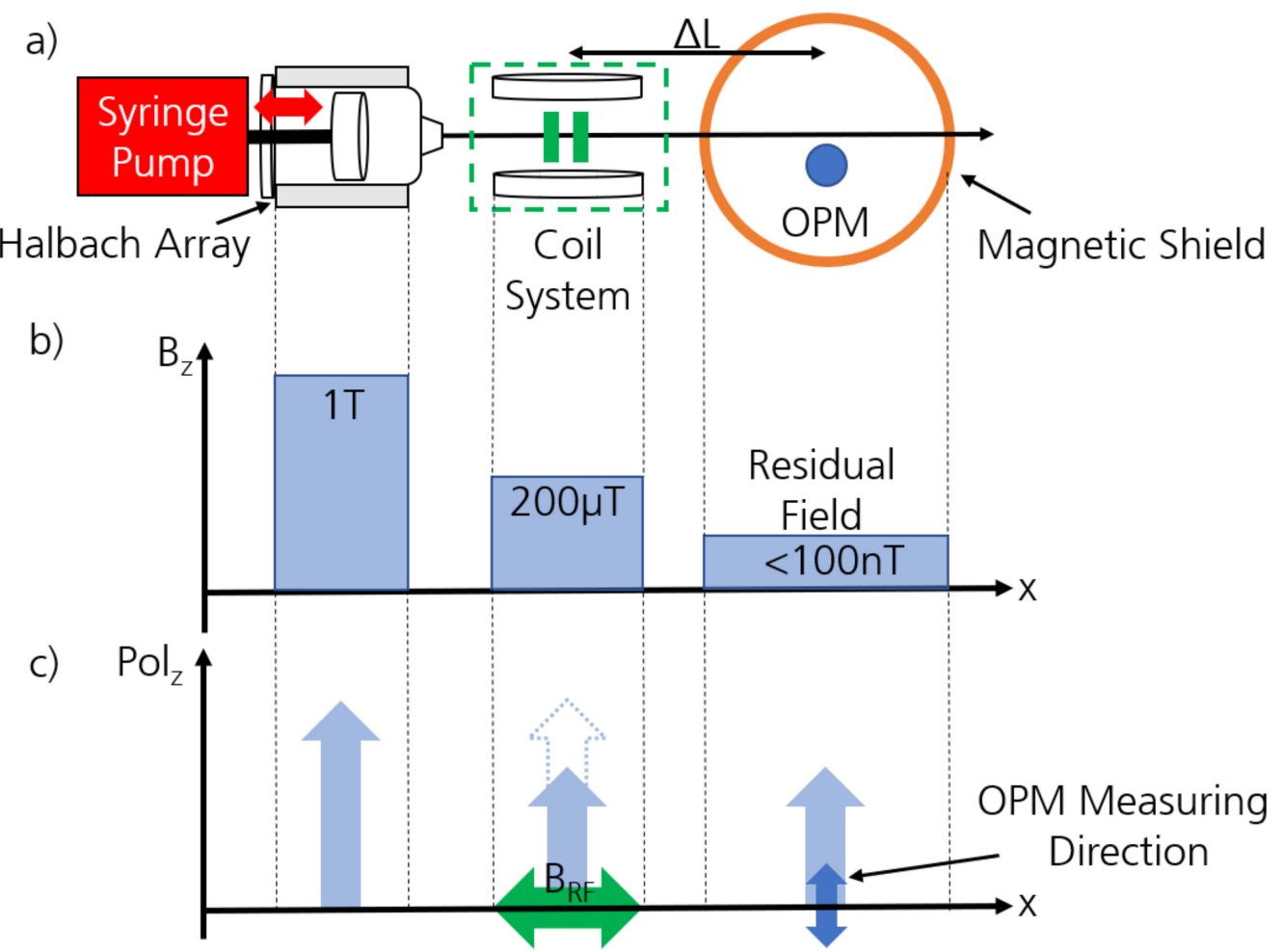

Figure 1. Schematic overview of the experimental setup with applied magnetic fields and the trend of the medium polarization. (a) Schematic of the experimental setup: The dashed green line encircles the interaction section with the coil system. The green square in the center marks the location of the RF coil. The two black cylinders represent the racetrack coils. The distance $\Delta \mathrm{L}$ between the center of the RF coil and the OPM is known and used to determine the flow velocity. The red double arrows indicate the direction of the pumping mechanism. (b) Orientation and field strengths of the magnetic fields as they are found in the apparatus. The magnetic field of the Halbach array to pre-polarize the water and the field of the racetrack coils point in z-direction. (c) Trend of the macroscopic water polarization throughout the water circuit in $\mathrm{z}$ direction. The RF coil operates in $\mathrm{x}$ direction to reorient the water polarization away from its pre-polarization direction. The OPM sensors measure the $z$ component of the field to detect how much water polarization is reoriented by the RF coil. The partial decay of the polarization amplitude during the flow of the medium through the tubing is neglected for simplicity.

The magnetic shield downstream contained zero field OPM for the detection of polarization changes induced by the RF coil. OPM are very sensitive sensors that can reach a sensitivity of $10 \mathrm{fT} / \sqrt{\mathrm{Hz}}$ [13] if they are operated in an environment where magnetic noise and the absolute magnetic field are strongly suppressed.

The distance $\Delta L$ between the center of the interaction section and the OPM was fixed at 212(1) $\mathrm{mm}$ throughout the experiment. A plastic tube with an inner diameter of $5 \mathrm{~mm}$ was used as a water guide. For validation, the data taken were compared to the pump rates indicated by the syringe pump in the end. Therefore, the flow velocity detected by the apparatus was converted to volumetric flow. 


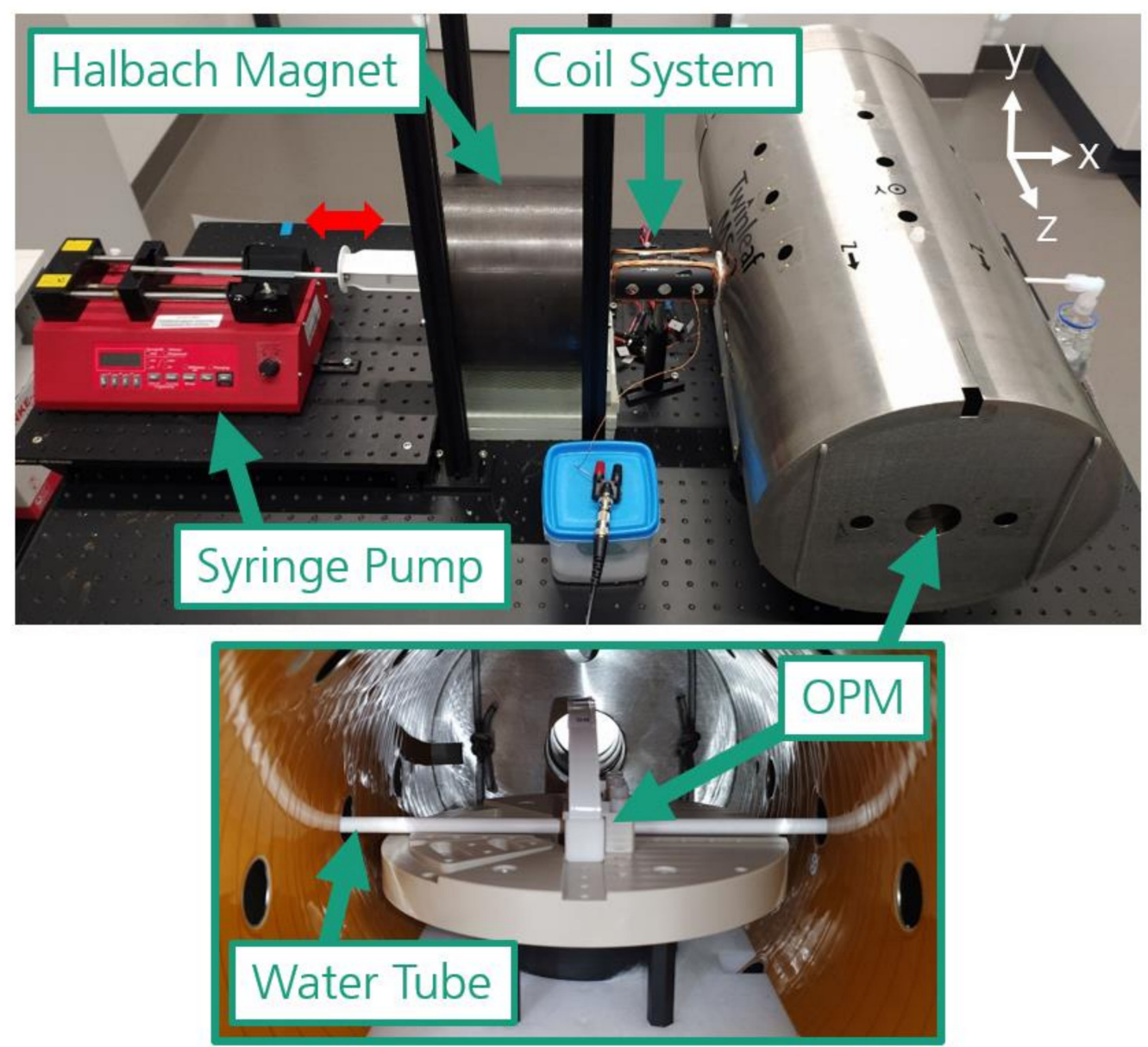

Figure 2. Image of the experimental setup with the syringe pump, the Halbach magnet with the syringe inside, the interaction section with the coil system and the magnetic shield containing the OPM sensors. The spatial directions are defined as indicated by the white arrows in the upper right corner. The interior of the magnetic shield is shown below. The white plastic pipe contains the water to be measured. The water crosses the image from left to right. The white OPM sensors are installed on a nonmagnetic mount in the center of the image. The mount enables accurate position referencing of the sensors. The sensitive axis of the sensors is in $\mathrm{z}$ direction. The red double arrow indicates the direction of the pumping mechanism.

\subsection{Procedure}

After starting the pump, the pre-polarized water flows through the apparatus. The water polarization interacts with the RF pulses of the RF coil in the interaction section (cf. Figure 3). This coil is operated with amplitude modulation (AM). The $8 \mathrm{kHz}$ RF signal has a sinusoidal envelope of $1 \mathrm{~V}$ at $100 \mathrm{mHz}$. The RF pulses manipulate the water polarization with an intensity proportional to the magnetic field generated by the AM RF coil. Thus, the RF coil periodically changes the polarization amplitude of the water passing the interaction section. Finally, the OPM detects an alternating magnetic amplitude of the water polarization analogue to the RF envelope. The time difference $\Delta t$ between pulse application and detecting its effect on the water polarization yields the time information to calculate the flow velocity $v$ using the distance $\Delta L$ :

$$
v=\frac{\Delta L}{\Delta t}
$$




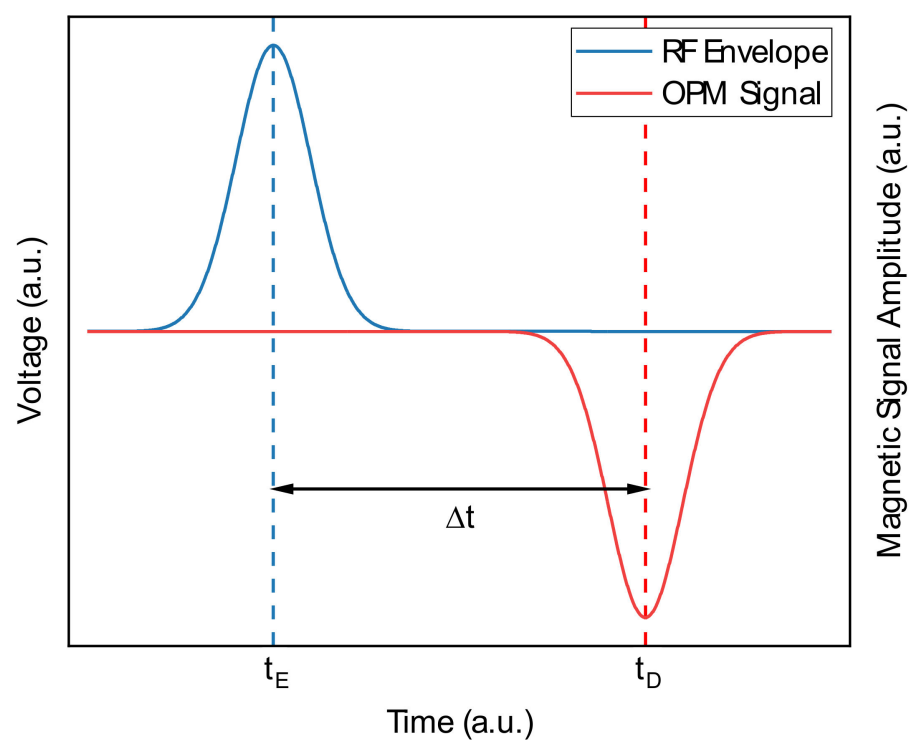

Figure 3. Schematic representation of the RF-Pulse and the OPM signal used in the experiment. The center of the RF pulse envelope (blue) defines the time $t_{E}$. The time where the OPM signal (red) reaches its minimum defines $t_{D}$. The quantity of interest is the delay between $t_{E}$ and $t_{D}: \Delta t=t_{D}-t_{E}$.

To compare the measured flow rate to the pump rates indicated by the pump, $v$ was converted to the average volumetric flow $Q$ inside the tube using the tube cross-section $A$ and its diameter $d$.

$$
Q=v A=v\left(\frac{d}{2}\right)^{2} \pi
$$

\subsection{Determination of $\Delta t$}

The voltage $U(t)$ applied to the RF coil is a sinusoid

$$
U(t)=A(t) \sin \left(\omega_{L} t\right)
$$

where $\omega_{L}$ is the Larmor frequency and $A(t)$ is the modulated amplitude with a sinusoidal shape. To feed the RF coil, only the positive halfwave was used. Let the time of excitation $t_{E}$ be defined as $A\left(t_{E}\right)=\max (A(t))$. Then the detection time $t_{D}$ is the corresponding point in time where the magnetic signal detected by the OPM is at its local minimum. An illustration of how the envelope RF voltage affects the magnetic signal recorded by the OPM is shown in Figure 3 . To determine $t_{E}$ and $t_{D}$ from the RF voltage and magnetic field data, parabolas were fitted to the raw data:

$$
f(t)=y\left(t-t_{E, D}\right)^{2}+\text { Offset. }
$$

The horizontal shift of the function $t_{E}$ and $t_{D}$ were used to calculate $\Delta t$ and determined as fit parameters. Thus, $\Delta t$ is the time difference between $t_{E}$ and $t_{D}$ :

$$
\Delta t=t_{D}-t_{E}
$$

This method of data acquisition implies that we measure the average flow of water inside the tube. For demonstration purposes the AM frequency was set to $100 \mathrm{mHz}$. 


\section{Results}

\subsection{Volumetric Flow Measurement}

Pump rates were varied from $150 \mathrm{~mL} / \mathrm{min}$ to $275 \mathrm{~mL} / \mathrm{min}$ in steps of $25 \mathrm{~mL} / \mathrm{min}$. According to Equation (2) these pump rates correspond to flow velocities from $12.7 \mathrm{~cm} / \mathrm{s}$ to $23.3 \mathrm{~cm} / \mathrm{s}$. For this velocity range the maximum Reynolds Number $R e$ is

$$
\operatorname{Re}=\frac{v d \rho}{\eta}=1165
$$

where $d$ is the tube diameter, $\rho$ is the density of water at $20^{\circ} \mathrm{C}$ and $\eta$ is the dynamic viscosity of water at $20^{\circ} \mathrm{C}$. Thus, $R e$ being smaller than $R e_{\text {crit }}=2300$, we assume that we have laminar flow. Hence, the apparatus measures the laminar flow of water inside the tube.

For each pump rate the syringe was emptied completely. The batch volume and the set pump rate determine the measuring time. In Figure 4 streamed data from the $200 \mathrm{~mL} / \mathrm{min}$ measurement are shown as an example. The OPM data were periodically modulated by the RF pulses and yielded a signal amplitude of about 60 pT. Please note that the Signal amplitude off-set was corrected in such a way that it was set to 0 before the start of the measurement.

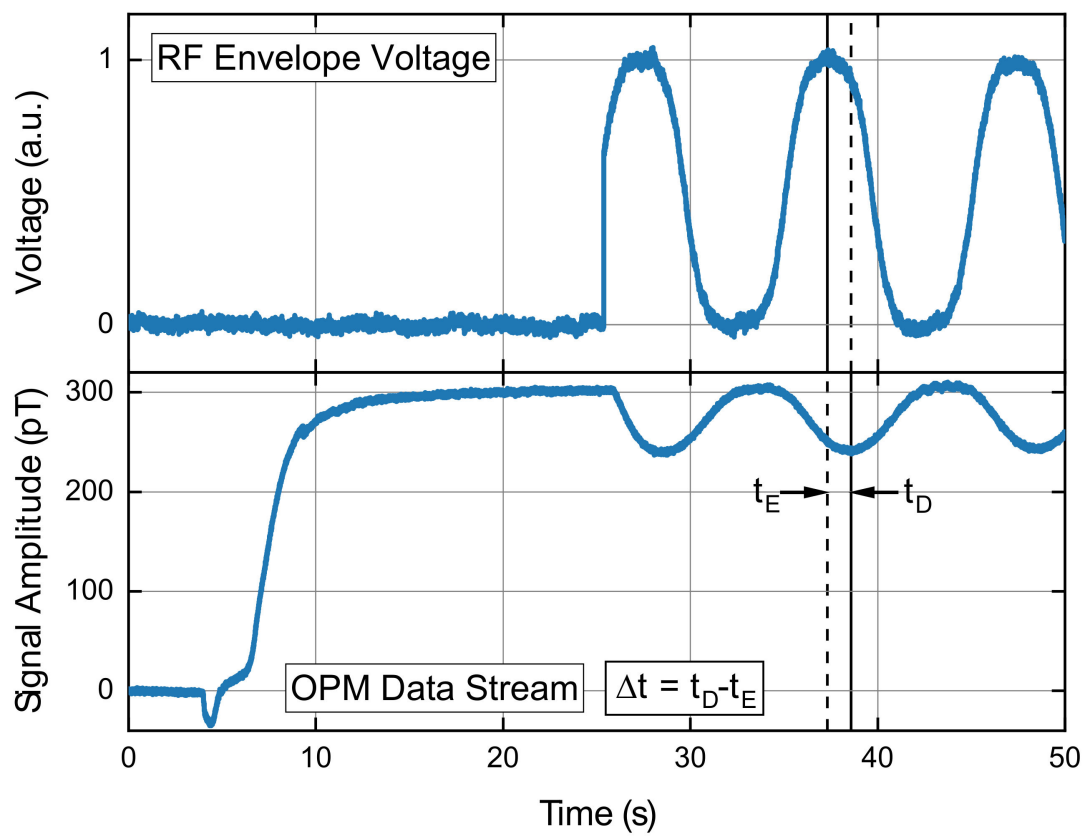

Figure 4. Data stream showing all relevant parameters for a flow measurement. The water velocity is $200 \mathrm{~mL} / \mathrm{min}$. The pump is turned on at $5 \mathrm{~s}$. Then, the polarized water flows past the OPM. This increases the magnetic field measured to a plateau region of 300 pT. At $25 \mathrm{~s}$ the RF coil is switched on, which periodically changes the water pre-polarization amplitude by about $60 \mathrm{pT}$. As explained in Figure 1, the OPM measures the vector component of the water polarization in $\mathrm{z}$ direction. Thus, it detects how much of the water pre-polarization is destroyed by the RF pulse. Residual fields in the shielded environment are on the order of nT. For this reason, the data are shifted to start at zero. Please note: The reference voltage plotted in the upper channel was recorded using the "trigger input" of the FieldLine OPM interface. The resulting line shape does not exactly reproduce the applied sinusoidal envelope of the RF pules. It is only used to determine $t_{E}$.

The flow rate and volumetric flow calculation was done as explained in Equations (1) and (2). For each pump rate, data were recorded until the syringe was completely emptied. Depending on the flow rate, every measurement yielded 5 to 14 data points. The flow velocity for a given pump rate was determined by the average of the individual values $t_{D}-t_{E}$ in one recording. The error on the average flow velocity was calculated from the scatter of the individual measured values for each pump rate. The results are listed in 
Table 1 and shown in Figure 5. On average, the relative error on the volumetric flow $Q$ and thus on the detected flow velocity $v$ was $3 \%$. In addition, the predicted pump rate of the syringe pump was mostly within one standard deviation of the detected volumetric flow. For $275 \mathrm{~mL} / \mathrm{min}$ the predicted pump rate lays within two standard deviations.

Table 1. Flow velocities and volumetric flows detected by the flow-metering apparatus. For comparison the pump rates of the syringe pump are listed in the left column. The manufacturer of the syringe pump noted an error of $<1 \%$ on the pump rate. The error specifications represent one standard deviation.

\begin{tabular}{ccc}
\hline Set Pump Rate $(\mathbf{m L} / \mathbf{m i n})$ & Flow Velocity $\mathbf{( c m / s )}$ & Calculated Volumetric Flow $(\mathbf{m L} / \mathbf{m i n})$ \\
\hline 150 & $13.0(3)$ & $153(4)$ \\
175 & $14.3(6)$ & $168(7)$ \\
200 & $17.4(5)$ & $205(6)$ \\
225 & $19.2(3)$ & $226(4)$ \\
250 & $20.9(8)$ & $246(9)$ \\
275 & $22.4(5)$ & $264(6)$ \\
\hline
\end{tabular}

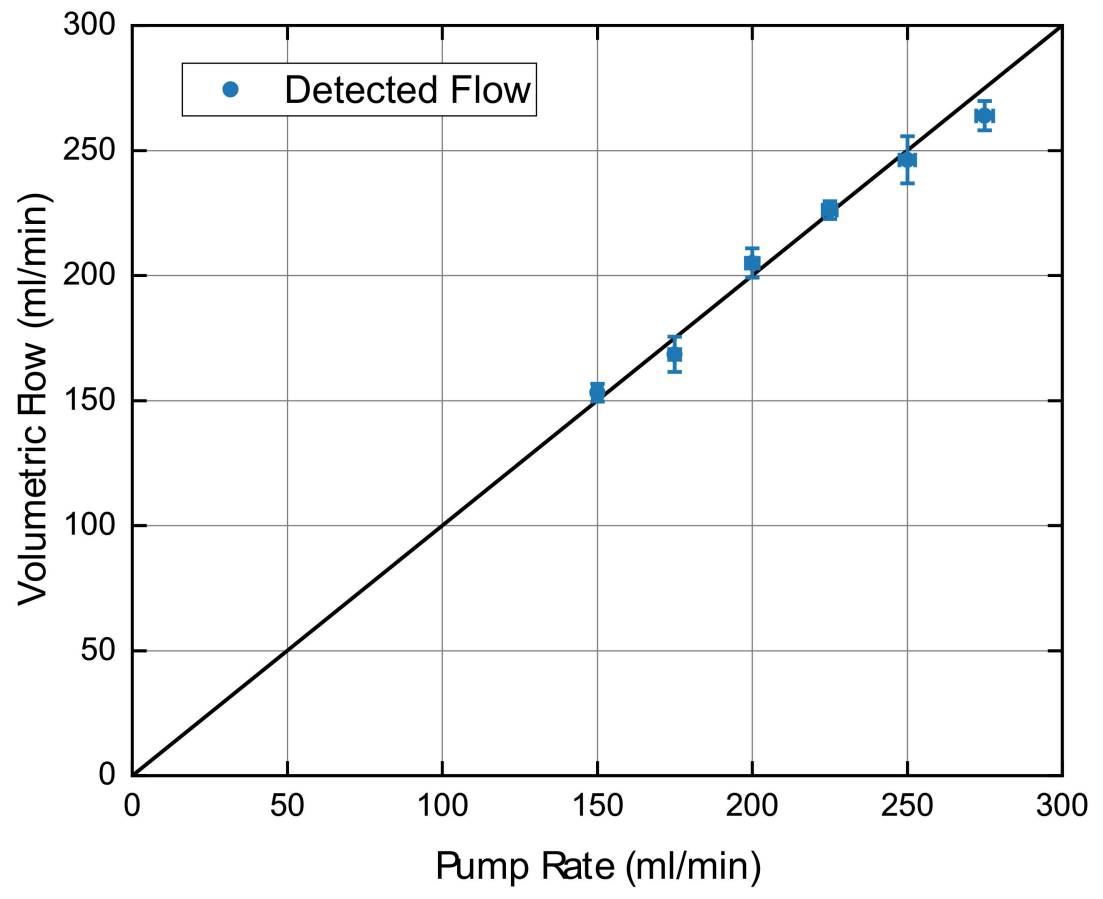

Figure 5. Comparison of the pump rate indicated by the syringe pump and the detected volumetric flow measured by the flow metering apparatus (blue). Error bars indicate one standard deviation. The black line indicates the ideal behavior. The error in $\mathrm{x}$-direction is set to $1 \%$ (pump specification: $<1 \%$ inaccuracy of the pump rate).

For any practical application of the presented scheme, the dynamic range of the procedure was limited by two factors. The first limitation derived from the decay of the medium polarization. For too-small flow velocities, the signal would decay before it arrived in front of the sensor. The second limitation was caused by bandwidth of the OPM sensor (currently $100 \mathrm{~Hz}$ ). This limited the accuracy of the determination of $t_{D}$ and, hence, of $\Delta t$ and the flow velocity.

\subsection{Short RF Pulsing}

In the experiments described above, the sinusoidal AM modulation of the RF pulses with $0.1 \mathrm{~Hz}$ limited both the data rate (one data point every $10 \mathrm{~s}$ ) and the accuracy of the determination of $\Delta t=t_{D}-t_{E}$. Shorter RF pulses are expected to produce narrower 
transients in the polarization signal, allowing one to determine $\Delta t$ more accurately and to increase the pulse rate.

In the following, an experiment was conducted as described in Section 2.2 but rectangular RF pulses with a duration of $250 \mathrm{~ms}$ and a repetition rate of $0.4 \mathrm{~Hz}$ were applied. The flow velocity was set to of $12.7 \mathrm{~cm} / \mathrm{s}$. For comparison, the amplitude modulated RF pulse approach has a pulse length of $5 \mathrm{~s}$. The RF amplitude in the experiment was $236 \mathrm{mV}$.

The experimental results are shown in Figure 6. The mean full width at half-maximum (FWHM) of the observed transients of the magnetic field was 0.38(4) s. The specified error was calculated as the standard deviation (1 sigma) of the scattering data.

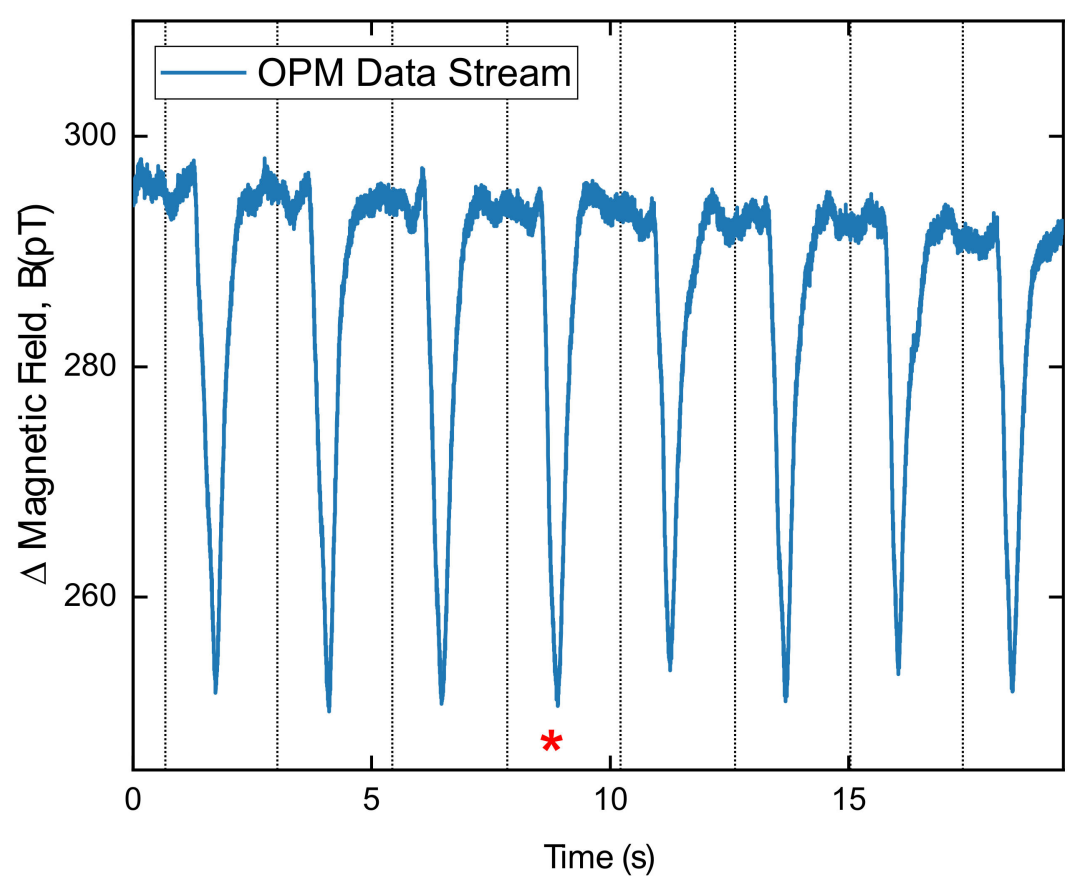

Figure 6. The experimental results for the application of short RF pulsing on the pre-polarized water. The black dotted lines mark the timing of the individual RF pulses. The asterisk marks the peak, which is used to compare the experimental data in Figure 7 with a signal estimate.

This width of the observed B field "pulses" was about 50\% larger than the duration of the applied RF pulses $(0.25 \mathrm{~s})$. To get a first insight into the action of the RF pulses on the flowing water, a simple model was used: The change of macroscopic polarization for a pre-polarized water voxel was assumed to be proportional to the integral $\int B d t$ experienced by the water voxel. Let $v$ be the velocity of the water inside the tube. Then the magnetic field experienced by a water voxel $S$ starting to move at a point $x_{0}$ inside the interaction section is given by

$$
S\left(x_{0}\right)=\int_{0}^{\text {Pulse Length }} B(x(t)) d t[T s],
$$

where $x(t)=x_{0}+v t$ is the position of the water voxel and $B(x(t))$ is the magnetic field at this position. In a first approximation, $B$ is calculated using the Biot-Savart approach for the magnetic field along the symmetric axis of the Helmholtz coils:

$$
B(x)=\frac{\mu_{0} N I}{2}\left(\frac{R^{2}}{\left(R^{2}+\left(x-\frac{d}{2}\right)^{2}\right)^{\frac{3}{2}}}+\frac{R^{2}}{\left(R^{2}+\left(x+\frac{d}{2}\right)^{2}\right)^{\frac{3}{2}}}\right)
$$

$\mu_{0}$ is the vacuum permeability, $N$ the number of coil windings of each coil, $I$ the electric current created by the RF voltage, $R$ the coil radius and $d$ the distance between 
the coils. $B(x)$ is shown in Figure 7 for a range of $\pm 10 \mathrm{~cm}$ from the coil center. Due to the geometric situation of the setup, the outer layer of the magnetic shield has approximately $6 \mathrm{~cm}$ from the center of the Helmholtz coil (see the shaded area in Figure 7). For this reason, the $B$ field distribution according to (8) shall only be considered a simple approximation.

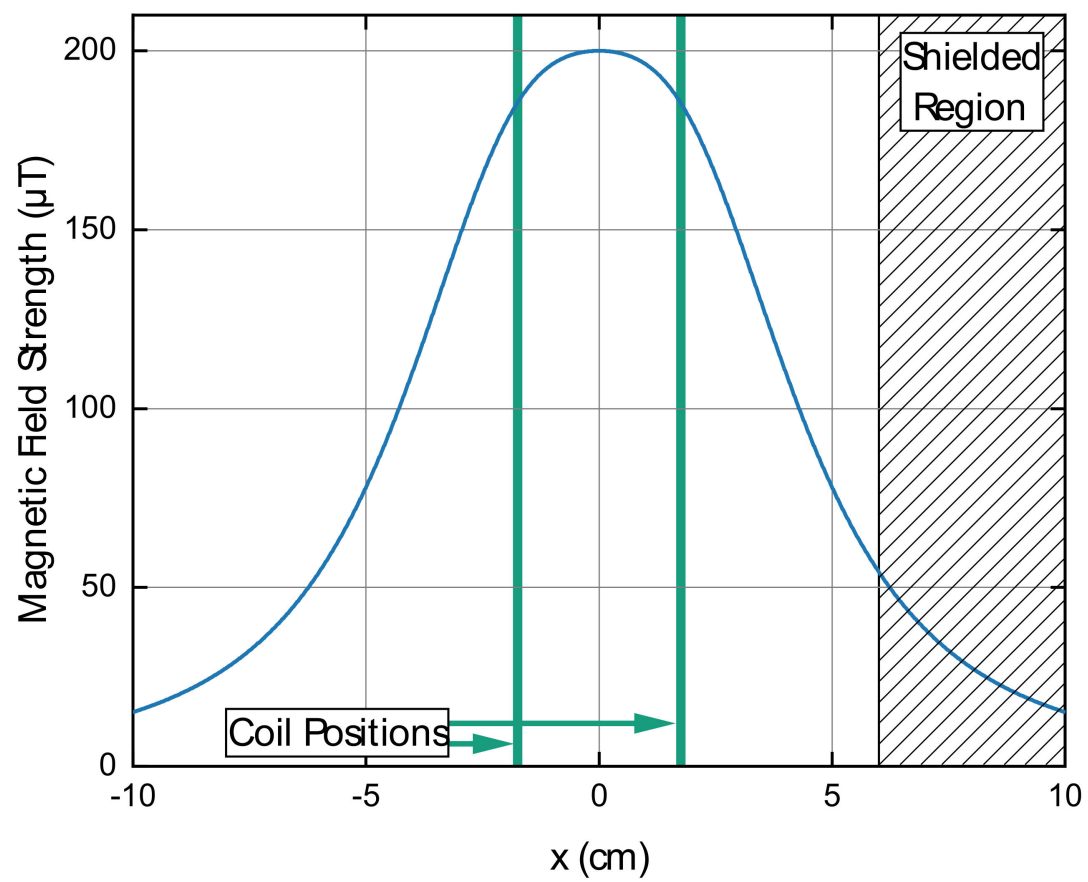

Figure 7. Spatial distribution of the magnetic field of the Helmholtz coil according to Equation (8). The distribution is calculated using a current of $200 \mathrm{~mA}$. The green lines mark the positions of the Helmholtz coil pair. The outermost layer of the magnetic shield is positioned at $x=6 \mathrm{~cm}$. As a consequence, the field distribution in the hatched region will differ from the simple model.

To make the result of $S\left(x_{0}\right)$ comparable to experimental OPM data, the spatial distribution was converted to a temporal distribution $S(t)$. This was done by converting the starting point argument $x_{0}$ to the time $t$ it takes for the water to arrive in front of the OPM sensor: $t=\left(\Delta L-x_{0}\right) / v . \Delta L$ is the distance between the RF coil and the OPM (cf. Figure 1).

The resulting expected signal according to (7) and an extract from the experimental data are shown in Figure 8 for comparison. For both curves the $\mathrm{x}$ axis was centered at $\mathrm{t}=0$. The y axis of the modeled signal was rescaled to match the peak height of the observed pulse. While the observed polarization "pulse width" (FWHM = $0.34 \mathrm{~s})$ was longer than the duration of the applied RF pulse ( $0.25 \mathrm{~s})$, it was clearly shorter than the width derived from our simple model $(0.67 \mathrm{~s})$. This, as well as the asymmetry of the observed "pulse" shape, indicates that the assumed $B$ field distribution along the $x$ axis may considerably differ from our simple model assumption. 


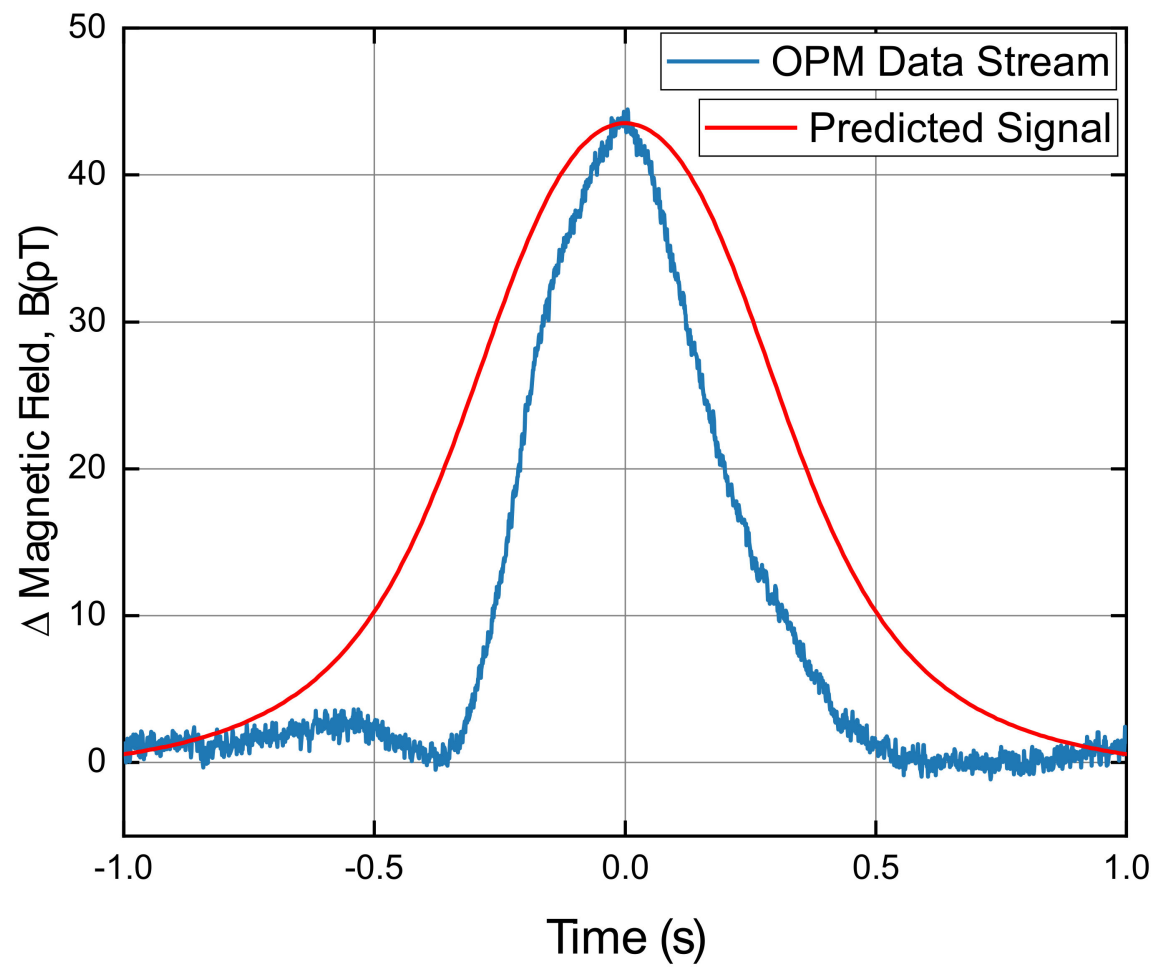

Figure 8. Extract of the experimental results with the predicted signal for comparison. The OPM data shows the peak of Figure 6 highlighted with a red asterisk. The predicted signal is longer than the experimental data and has a full width at half-maximum (FWHM) of $0.67 \mathrm{~s}$. The experimental data has a FWHM of $0.39 \mathrm{~s}$. In a first approximation, we assumed the magnetic field of the RF coil is symmetric, which is not confirmed by the data. Thus, we refrain from a detailed analysis.

\section{Conclusions}

We presented a noninvasive, magnetic-marking-based flow metering procedure, which uses an OPM operating in the ZULF regime at magnetic-field strengths lower than $100 \mathrm{nT}$ as the signal detector. The detection method was based on a time-of-flight measurement, which makes the procedure calibration-free.

In the frame of the experiments, a macroscopic polarization of $300 \mathrm{pT}$ was created in the water samples using a $1 \mathrm{~T}$ Halbach magnet. Applying short RF pulses as "markers" on the water flow resulted in polarization transients with amplitudes between 10 and $80 \mathrm{pT}$, which were detected with the OPM with good signal-to-noise ratio and used to derive the flow velocity of the medium.

In the current demonstration setup, the useful flow velocities ranged between $12.7 \mathrm{~cm} / \mathrm{s}$ and $23.3 \mathrm{~cm} / \mathrm{s}$. Utilizing the procedure presented, we successfully demonstrated measuring the water flow with an average accuracy of $3 \%$ in the given velocity interval. Comparing the measured results to the pump rates of the syringe pump showed that the data were consistent with expected values.

The current experiments demonstrate the feasibility of a marker-free magnetometrybased flow metering, enabled by the outstanding performance of commercially available optically pumped magnetometers. A number of steps will be necessary to move from our "proof-of-concept" experiment to a metering system applicable in practical situations by answering the following questions:

What signals are still detectable in a certain background situation? How much shielding effort is required? Which level of pre-polarization and RF pulsing is required? What is the resulting time accuracy? What RF pulse form is best suited?

The mentioned aspects must not be considered isolated from each other. Addressing them is the topic of experiments currently under way. 
Author Contributions: L.S., P.A.K. and A.L. conceptualized the research. L.S. conceived the initial idea of the procedure, performed the experiments, and wrote a draft version of the manuscript. P.A.K. and L.S. designed the experimental setup. All authors analyzed the data and revised the final version of the manuscript. All authors have read and agreed to the published version of the manuscript.

Funding: This work was supported as a Fraunhofer LIGHTHOUSE PROJECT (QMag). In equal parts, this work was funded by the Ministry of Economic Affairs, Labour and Housing of the State of Baden-Württemberg, Germany.

Data Availability Statement: The data is available from the corresponding author upon reasonable request.

Acknowledgments: We acknowledge Michael Bock of the Medical Centre of Freiburg, Germany for his helpful discussions on this research.

Conflicts of Interest: The authors declare no conflict of interest.

\section{References}

1. Endress + Hauser. Flow Measuring Technology for Liquids, Gases and Steam. Available online: https://portal.endress.com/wa0 01/dla/5000192/0856/000/02/FA00005DEN_1918.pdf (accessed on 30 November 2021).

2. $\quad$ Bilgic, A.M.; Kunze, J.W.; Stegemann, V.; Zoeteweij, M.; Hogendoorn, J. (Eds.) Multiphase Flow Metering with Nuclear Magnetic Resonance Spectroscopy; AMA Service GmbH: Wunstorf, Germany, 2015; p. 6.

3. Thorn, R.; Johansen, G.A.; Hjertaker, B.T. Three-phase flow measurement in the petroleum industry. Meas. Sci. Technol. 2013, 24, 12003. [CrossRef]

4. Halbach, R.E.; Salles-Cunha, S.; Battocletti, J.H.; Sances, A.; Bernhard, V.M. Noninvasive measurement of arterial blood flow by means of a nuclear magnetic resonance flowmeter. Surg. Forum 1978, 29, 220-222.

5. Caprihan, A.; Fukushima, E. Flow Measurements by NMR. Phys. Rep. 1990, 198, 195-235.

6. Falcone, G.; Hewitt, G.F.; Alimonti, C.; Harrison, B. Multiphase flow metering: Current trends and future developments. J. Pet. Technol. 2002, 54, 77-84.

7. Kraus, R.H.; Espy, M.A.; Magnelind, P.E.; Volegov, P.L. Ultra-Low Field Nuclear Magnetic Resonance: A New MRI Regime; Oxford University Press: New York, NY, USA, 2014; ISBN 978-0-19-979643-4.

8. Ganssle, P.J.; Shin, H.D.; Seltzer, S.J.; Bajaj, V.S.; Ledbetter, M.P.; Budker, D.; Knappe, S.; Kitching, J.; Pines, A. Ultra-Low-Field NMR Relaxation and Diffusion Measurements Using an Optical Magnetometer. Angew. Chem. Int. Ed Engl. 2014, 53, 9766-9770. [CrossRef]

9. Fridjonsson, E.O.; Creber, S.A.; Vrouwenvelder, J.S.; Johns, M.L. Magnetic resonance signal moment determination using the Earth's magnetic field. J. Magn. Reson. 2015, 252, 145-150. [CrossRef]

10. O'Neill, K.T.; Klotz, A.; Stanwix, P.L.; Fridjonsson, E.O.; Johns, M.L. Quantitative multiphase flow characterisation using an Earth's field NMR flow meter. Flow Meas. Instrum. 2017, 58, 104-111. [CrossRef]

11. Fridjonsson, E.O.; Stanwix, P.L.; Johns, M.L. Earth's field NMR flow meter: Preliminary quantitative measurements. J. Magn. Reson. 2014, 245, 110-115. [CrossRef]

12. Greenberg, Y.S. Application of superconducting quantum interference devices to nuclear magnetic resonance. Rev. Mod. Phys. 1998, 70, 175-222. [CrossRef]

13. Shah, V.K.; Wakai, R.T. A compact, high performance atomic magnetometer for biomedical applications. Phys. Med. Biol. 2013, 58, 8153-8161. [CrossRef]

14. Budker, D.; Romalis, M. Optical magnetometry. Nat. Phys. 2007, 3, 227-234. [CrossRef]

15. Guío-Pérez, D.C.; Dietrich, F.; Ferreira Cala, J.N.; Pröll, T.; Hofbauer, H. Estimation of solids circulation rate through magnetic tracer tests. Powder Technol. 2017, 316, 650-657. [CrossRef] 